\title{
RDF Data-Centric Storage
}

Justin J. Levandoski

Department of Computer Science and Engineering, University of Minnesota, Minneapolis, MN
Mohamed F. Mokbel

\{justin,mokbel@cs.umn.edu\}

\begin{abstract}
The vision of the Semantic Web has brought about new challenges at the intersection of web research and data management. One fundamental research issue at this intersection is the storage of the Resource Description Framework (RDF) data: the model at the core of the Semantic Web. We present a data-centric approach for storage of RDF in relational databases. The intuition behind our approach is that each RDF dataset requires a tailored table schema that achieves efficient query processing by (1) reducing the need for joins in the query plan and (2) keeping null storage below a given threshold. Using a basic structure derived from the RDF data, we propose a two-phase algorithm involving clustering and partitioning. The clustering phase aims to reduce the need for joins in a query. The partitioning phase aims to optimize storage of extra (i.e., null) data in the underlying relational database. Our approach does not assume a particular query workload, relevant for RDF knowledge bases with a large number of ad-hoc queries. Extensive experimental evidence using three publicly available real-world RDF data sets (i.e., DBLP, DBPedia, and Uniprot) shows that our schema creation technique provides superior query processing performance compared to state-of-the art storage approaches. Further, our approach is easily implemented, and complements existing RDF-specific databases.
\end{abstract}

\section{INTRODUCTION}

Over the past decade, the W3C [1] has led an effort to build the Semantic Web. The purpose of the Semantic Web is to provide a common framework for data-sharing across applications, enterprises, and communities [2]. By giving data semantic meaning (through metadata), this framework allows machines to consume, understand, and reason about the structure and purpose of the data. In this way, the Semantic Web resembles a worldwide database, where humans or computer agents can pose semantically meaningful queries and receive answers from a variety of distributed and distinct sources. The core of the Semantic Web is built on the Resource Description Framework (RDF) data model. RDF provides a simple syntax, where each data item is broken down into a $<$ subject, property, object> triple. The subject represents an entity instance, identified by a Uniform Resource Identifier (URI). The property represents an attribute of the entity, while the object represents the value of the property. As a simple example, the following RDF triples model the fact that a person John is a reviewer for the conference ICWS 2009:

$$
\begin{aligned}
& \text { person1 hasName ' John'' } \\
& \text { confICWS09 hasTitle ' 'ICWS 2009', } \\
& \text { person1 isReviewerFor confICWS09 }
\end{aligned}
$$

While the ubiquity of the RDF data model has yet to be realized, many application areas and use-cases exist for RDF, such as education [3], mobile search environments [4], social

This work is supported in part by the National Science Foundation under Grants IIS0811998, IIS0811935, and CNS0708604

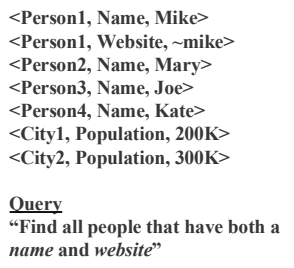

(a) RDF Triples

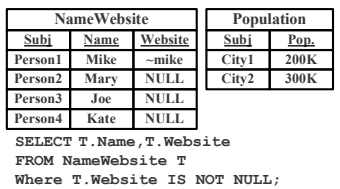

(c) $\mathrm{N}$-ary Table

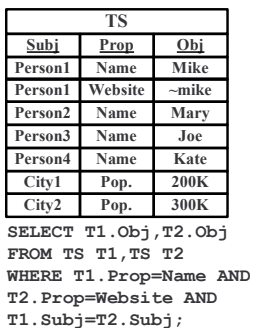

(b) Triple Store

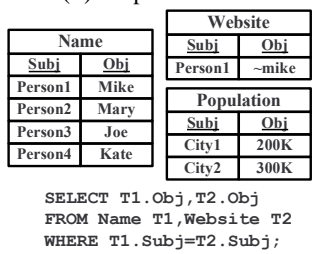

(d) Binary Tables
Fig. 1. RDF Storage Example

networking [5], and biology and life science [6], making it an emerging and challenging research domain.

Efficient and scalable management of RDF data is a fundamental challenge at the core of the Semantic Web. Many popular RDF storage solutions use relational databases to achieve this scalability and efficiency. To illustrate, Figure 1(a) gives a sample set of RDF triples for information about four people and two cities, along with a simple query that asks for people with both a name and website. Figures 1(b) - 1(d) give three possible approaches to storing these sample RDF triples in a DBMS, along with the translated RDF queries given in SQL. A large number of systems use a triple-store schema [7], [8], [9], [10], [11], [12], where each RDF triple is stored directly in a three-column table (Figure 1(b)). This approach provides inefficient query execution due to a proliferation of selfjoins, an expensive operation whereby the triple-store is joined against itself. Another approach is the property table [10], [13] (Figure 1(c)) that models multiple RDF properties as n-ary table columns. The $n$-ary table eliminates the need for a join in our example query. However, as only one person out of four has a website, the n-ary table contains a high number of nulls (i.e., the data is semi-structured), potentially causing a high overhead in query processing [14]. The decomposed storage schema [15] (Figure 1(d)) stores triples for each RDF property in a binary table. The binary table approach reduces null storage, but introduces a join in our example query.

In this paper, we propose a new storage solution for RDF data that aims to avoid the drawbacks of these previous approaches. Our approach is data-centric, as it tailors a relational schema based on a derived structure of the RDF data with 
the explicit goal of providing efficient query performance. We achieve this goal by taking into account the following trade-off of expensive query processing operations in relational databases: (1) reducing, on average, the need to join tables in a query by storing as much RDF data together as possible, and (2) reducing the need to process extra data by tuning extra storage (i.e., null storage) to fall below a given threshold. To handle these trade-offs, our approach involves two phases: clustering and partitioning. The clustering phase scans the RDF data to find groups of related properties (i.e., properties that always exist together for a large number of subjects). Properties in a cluster are candidates for storage together in an n-ary table. Likewise, properties not in a cluster are candidates for storage in binary tables. The partitioning phase takes clusters from the clustering phase and balances the tradeoff between storing as many RDF properties in clusters as possible while keeping null storage to a minimum (i.e., below a given threshold). Our approach also handles cases involving multi-valued properties (i.e., properties defined multiple times for a single subject) and reification (i.e., extra information attached to a whole RDF triple). The output of our schema creation approach can be considered a balanced mix of binary and n-ary tables based on the structure of the data.

The performance of our data-centric approach is backed by experiments on three large publicly available real-world RDF data sets: DBLP [16], DBPedia [17], and Uniprot [6]. Each of these data show a range of schema needs, and a set of benchmark queries are used to show that our datacentric schema creation approach improves query processing compared to previous approaches. Results show that our datacentric approach achieves orders of magnitude performance improvement over the triple store, and speedup factors of up to 36 over a straight binary table approach.

\section{RELATED WORK}

Previous approaches to RDF storage have focused on three main categories. (1) The triple-store (Figure 1(b)). Relational architectures that make use of a triple-store as their primary storage scheme include Oracle [10], Sesame [9], 3-Store [11], R-Star [12], RDFSuite [7], and Redland [8]. (2) The property table (Figure 1(c)). Due to the proliferations of self-joins involved with the triple-store, the property table approach was proposed. Architectures that make use of property tables as their primary storage scheme include the Jena Semantic Web Toolkit [13]. Oracle [10] also makes use of property tables as secondary structures, called materialized join views (MJVs). (3) The decomposed storage model [18] (Figure 1(d)) has recently been proposed as an RDF storage method [15], and has been shown to scale well on column-oriented databases, with mixed results for row-stores. Our work distinguishes itself from previous work as we (1) provide a tailored schema for each RDF data set, using a balance between n-ary tables (i.e., property tables) and binary tables (i.e.,decomposed storage), and (2) provide an automated method to place properties together in tables based on the structure of the data. Previous approaches to building property tables have involved the use of generic pre-computed joins, or construction by a DBA with knowledge of query usage statistics [10]. It is important to note, however, that our schema creation technique is systemindependent, and can complement any related full-fledged RDF-specific databases (e.g., see [7], [10], [8], [11]).

Other work in RDF storage has dealt with storing precomputed paths in a relational database [19], used to answer graph queries over the data (i.e., connection, shortest path). Other graph database approaches to RDF, including extensions to RDF query languages to support graph queries, have been proposed [20]. This work is outside the scope of this paper, as we do not study the effect of graph queries over RDF.

Automated relational schema design has primarily been studied with the assumption of query workload statistics. Techniques have been proposed for index and materialized view creation [21], horizontal and vertical partitioning [22], [23], and partitioning for large scientific workloads [24]. Our automated data-centric schema design method for RDF differs from these approaches in two main ways. (1) Our method does not assume a set of query workload statistics, rather, we base our method on the structure found in RDF data. We believe this is a reasonable assumption given the fact that queries over RDF knowledge bases tend to be ad-hoc. (2) Previous schema creation techniques cannot take into account the heterogeneous

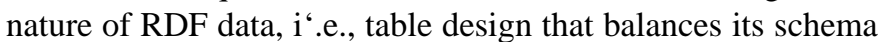
between well-structured and semi-structured data sets.

\section{Overview \& Problem Definition}

Overview. In general, two modules exist outside the database engine to handle RDF data and queries: (1) an RDF import module, and (2) an RDF query module. Our proposed data-centric schema creation technique exists inside the RDF import module. The schema creation process takes as input an RDF data set. The output of our technique is a schema (i.e., a set of relational tables) used to store the imported RDF data in the underlying DBMS.

Problem Definition. Given a data set of RDF triples, generate a relational table schema that achieves the following criteria. (1) Maximize the likelihood that queries will access properties in the same table without causing a join and (2) Minimize the amount of unnecessary data processing by reducing extra data storage (e.g., null storage).

Join operations along with extra table accesses produce a large query processing overhead in relational databases. Our schema creation method aims to achieve the first criterion by explicitly aiming to maximize the amount RDF data stored together in n-ary tables. However, as we saw in the example given in Figure 1, n-ary tables can lead to extra storage that affects query processing. Thus, our schema creation method aims to achieve the second criterion by keeping the null storage in each table below a given threshold.

\section{DAtA-CEntric SCHEMA CREATION}

The intuition behind our approach is that different RDF data sets require different storage structures. For example, a relatively well-structured RDF data set (i.e., data where the majority of relevant RDF properties are defined for the 


\begin{tabular}{|c|c|c|}
\hline Property & Usage & \multirow{3}{*}{$\begin{array}{|ll|}P C: \quad & \{\boldsymbol{P} 1, \boldsymbol{P} 2, \boldsymbol{P} 3, \boldsymbol{P} 4\} \text { (54\% Support) } \\
& \{\boldsymbol{P 1}, \boldsymbol{P} 2, \boldsymbol{P 5}, \boldsymbol{P} 6\} \text { (45\% Support) } \\
& \{\boldsymbol{P} 7, \boldsymbol{P} \boldsymbol{8}\} \text { (30\% Support) } \\
\end{array}$} \\
\hline P1 & 1000 & \\
\hline P2 & 500 & \\
\hline P3 & 700 & \multirow{3}{*}{$\begin{array}{l}\text { NullPercentage }(\{\boldsymbol{P} \mathbf{1}, \boldsymbol{P} 2, \boldsymbol{P} 3, \boldsymbol{P} 4\})=21 \% \\
\text { NullPercentage }(\{\boldsymbol{P} \mathbf{1}, \boldsymbol{P} \mathbf{2}, \boldsymbol{P} \mathbf{5}, \boldsymbol{P} \mathbf{6}\})=32 \% \\
\text { NullPercentage }(\{\boldsymbol{P} 7, \boldsymbol{P} \mathbf{8}\})=4 \% \\
\end{array}$} \\
\hline P4 & 750 & \\
\hline P5 & 450 & \\
\hline P6 & 450 & \multirow{2}{*}{$\begin{array}{l}\text { NullPercentage }(\{\boldsymbol{P} \mathbf{1}, \boldsymbol{P} \mathbf{3}, \boldsymbol{P} 4\})=13 \% \\
\text { NullPercentage }(\{\boldsymbol{P} 2, \boldsymbol{P} \mathbf{5}, \boldsymbol{P} \mathbf{6}\})=5 \%\end{array}$} \\
\hline P7 & 300 & \\
\hline P8 & 350 & \multirow{2}{*}{ 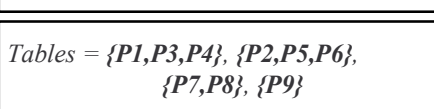 } \\
\hline P9 & 50 & \\
\hline
\end{tabular}

Fig. 2. RDF Data Partitioning Example

subjects) may require a few large n-ary tables. A semistructured data set (i.e., data that does not follow a fixed pattern for property definition) may use a large number of binary tables as its primary storage schema.

\section{A. Algorithm Overview and Data Structures}

Our schema creation algorithm takes as parameters an RDF data set, along with two numerical values: support threshold and null threshold. Support threshold is a value used to measure strength of correlation between properties in the RDF data. If a set of properties meets this threshold, they are candidates to exist in the same n-ary table. The null threshold is the percentage of null storage tolerated for each table in the schema.

The data structures for our algorithm are built using an $\mathrm{O}(n)$ process that scans the RDF triples once (where $n$ is the number of RDF triples). We maintain two data structures: (1) Property usage list. This is a list structure that stores, for each property defined in the RDF data set, the count of subjects that have that property defined. For example, if a property usage list were built for the data in Figure 1(a), the property Website would have a usage count of one, as it is only defined for the subject Person1. Likewise, the Name property would have a usage count of four, and Population would have a count of two. (2) Subject-property baskets. This is a list of all RDF subjects mapped to their associated properties (i.e., a property basket). A single entry in the subject-property basket structure takes the form $s u b j I d \rightarrow\left\{\right.$ prop $_{1}, \cdots$, prop $\left._{n}\right\}$, where $\operatorname{subjId}$ is the Uniform Resource Identifier of an RDF subject and its property basket is the list of all properties defined for that subject. As an example, for the sample data in Figure 1(a), six baskets would be created by this process: Person $1 \rightarrow\{$ Name, Website $\}$, Person $2 \rightarrow\{$ Name $\}$, Person $3 \rightarrow\{$ Name $\}$, Person $4 \rightarrow\{$ Name $\}$, City $1 \rightarrow\{$ Population $\}$, City $2 \rightarrow\{$ Population $\}$.

Our schema creation algorithm involves two phases: clustering and partitioning. The clustering phase (Phase I) aims to find groups of related properties in the data set using the support threshold parameter. Clustering leverages previous work from association rule mining to find related properties in the data. The idea behind the clustering phase is that properties contained in the clusters should be stored in the same n-ary table. The canonical argument for n-ary tables is that related properties are likely to be queried together. Thus, storing related properties together in a single table will reduce the number of joins during query execution. The clustering phase also creates an initial set of final tables. These initial tables consist of the properties that are not found in the generated clusters (thus being stored in binary tables) and the property clusters that do not need partitioning (i.e., in Phase II). The partitioning phase (Phase II) takes the clusters from Phase I and ensures that they contain a disjoint set of properties while keeping the null storage for each cluster below a given threshold. The final schema is the union of tables created from Phase I and II.

\section{B. Phase I: Clustering}

The clustering phase involves two steps. Step 1: A set of clusters (i.e., related properties) are found by leveraging the use of frequent itemset finding, a method used in association rule mining [25]. For our purposes, the terms frequent itemsets and clusters are used synonymously. The clustering phase finds groups of properties that are found often in the subjectproperty basket data structure. The measure of how often a cluster occurs is called its support. Clusters with high support imply many RDF subjects have all of the properties in the cluster defined. In other words, high support implies that properties in a cluster are related since they often exist together in the data. The metric for high support is set by the support threshold parameter to our algorithm, meaning we consider a group of properties to be a cluster only if they have support greater than or equal to the support threshold. If we specify a high support threshold, the clustering phase will produce a small number of small clusters with highly correlated properties. For low support threshold, the clustering phase will produce a greater number of large clusters, with less-correlated properties. Also, for our purposes, we are only concerned with maximum sized cluster (or maximum frequent itemsets); these are the clusters that occur often in the data and contain the most properties, meaning we maximize the data stored in n-ary tables. It is important to note that maximum frequent itemset generation can produce clusters with overlapping properties.

Step 2: Construct an initial set of final tables. These tables contain (1) properties that are not found in generated clusters (thus being stored in binary tables) and (2) the property clusters that meet the null threshold and do not contain properties that overlap with other clusters, thus not necessitating Phase II. Clusters that are added to the initial final table list are removed from the cluster list. The output of the clustering phase is a list of initial final tables, and a set of clusters, sorted in decreasing order by their support value, that will be sent to the partitioning phase.

Example. Consider an example with a support threshold of $15 \%$, null threshold of $20 \%$ for the six subject-property baskets given in Section IV-A for the data in Figure 1(a). In this case we have four possible property clusters: $\{$ Name $\},\{$ Website $\}$, $\{$ Population $\}$, and $\{$ Name, Website $\}$. The cluster $\{$ Name $\}$ occurs in 4 of the 6 property baskets, giving it a support of $66 \%$, while the cluster $\{$ Name, Website $\}$ occurs in 1 of 6 property baskets, giving it a support of $16 \%$. In this case, the $\{$ Name, Website $\}$ is generated as a cluster, since 


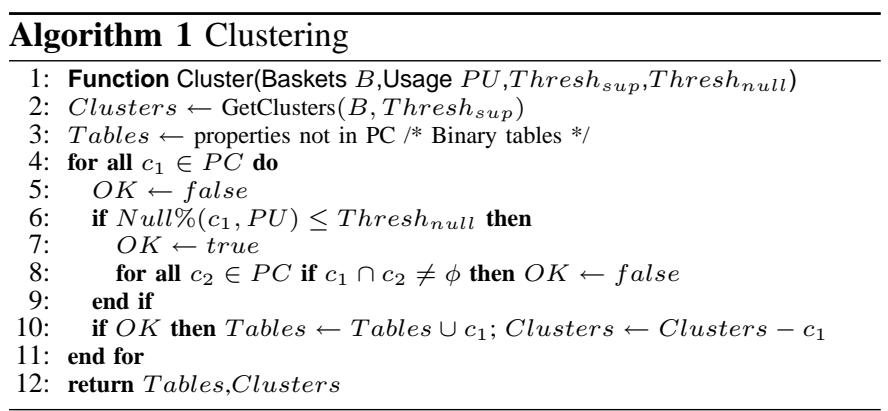

it meets the support threshold and has the most possible properties. Note the single property $\{$ Population\} is not considered a cluster, and would be added to the initial final table list. Also, Figure 2(b) gives three example clusters along with their support values, while Figure 2 (c) gives their null storage values (null storage calculation is covered shortly). The output of the clustering phase in this example with a support and null threshold value of $20 \%$ would produce an initial final table list containing $\{P 9\}$ (not contained in a a cluster) and $\{P 7, P 8\}$ (not containing overlapping properties and meeting the null threshold). The set of output clusters to be sent to the next phase would contain $\{P 1, P 2, P 3, P 4\}$ and $\{P 1, P 2, P 5, P 6\}$.

Algorithm. Algorithm 1 gives the pseudocode for the clustering phase, and takes as parameters the subject-property baskets $(B)$, the property usage list $(P U)$, the support threshold $\left(\right.$ Thresh $\left._{\text {sup }}\right)$, and the null threshold parameter $\left(\right.$ Thresh $\left._{\text {null }}\right)$. Step 1 of the algorithm generates clusters, sorts them by support value, and stores them in list Clusters (Line 1 in Algorithm 1). This is a direct call to a maximum frequent itemset algorithm [25], [26]. Step 2 of the algorithm is performed by first setting a list Tables to all binary tables returned from step 1. Tables is then expanded by adding from list Clusters the clusters that do not contain overlapping properties that are below the given null threshold (Lines 1 to 1 in Algorithm 1). Calculation of the null storage for a cluster $c$ is performed using the property usage list $P U$. Let $|c|$ be the number of properties in a cluster, and PU.maxcount $(c)$ be the maximum property count for a cluster in $P U$. As an example, in Figure 2 (b) if $c=\{P 1, P 2, P 3, P 4\}),|c|=4$ and PU.maxcount $(c)=1000$ (corresponding to $P 1$ ). If $P U$.count $\left(c_{i}\right)$ is the usage count for the $i$ th property in $c$, the null storage percentage for $c$ is:

$$
N u l l \%(c)=\frac{\sum_{\forall i \in c}\left(P U . \operatorname{maxcount}(c)-P U . \operatorname{count}\left(c_{i}\right)\right)}{(|c|+1) * P U \cdot \operatorname{maxcount}(c)}
$$

Finally, the algorithm returns the initial final table list and remaining clusters (Line 1 in Algorithm 1).

\section{Phase II: Partitioning}

The objective of the partitioning phase is twofold: (1) Partitioning the given clusters (from Phase I) into a set of non-overlapping clusters (i.e., a property exists in a single n-ary table). Ensuring that a property exists in a single cluster reduces the number of table accesses and unions necessary in query processing. For example, consider two possible n-ary tables storing RDF data for publications: TitleConf $=\{$ subj,title, conference $\}$ and TitleJourn $=$ $\{s u b j$, title, journal $\}$. An RDF query asking for all published titles would involve two table accesses and a union, since titles exist in both the conference and journal tables. (2) Ensuring that each partitioned cluster, falls below the null storage threshold. Reducing null storage tunes the schema for efficient query processing.

We propose a greedy algorithm that attempts to keep the cluster with highest support intact, while pruning lowersupport clusters containing overlapping properties. The intuition behind the greedy approach is that clusters with highest support contain properties that occur together most often in the RDF data. Support is the percentage of RDF subjects that have all of the cluster's properties. Keeping high support clusters intact implies that the most RDF subjects (with the cluster's properties defined) will be stored together in the table. Our greedy approach continually considers the highest support cluster, and handles two cases based on its null storage computation (from Section IV-B). Case 1: the cluster meets the null storage threshold, meaning the given cluster from Phase I meets the null threshold but contains overlapping properties. In this case, the cluster is considered a table and all lower-support clusters with overlapping properties are pruned (i.e., the overlapping properties are removed from these lowersupport clusters). We note that pruning will likely create overlapping cluster fragments; these are clusters that are no longer maximum sized (i.e., maximum frequent itemsets) and contain similar properties. To illustrate, consider a list of three clusters $c_{1}=\{A, B, C, D\}, c_{2}=\{A, B, E, F\}$, and $c_{3}=\{C, E\}$ such that support $\left(c_{1}\right)>\operatorname{support}\left(c_{2}\right)>\operatorname{support}\left(c_{3}\right)$. Since our greedy approach chooses $c_{1}$ as a final table, pruning creates overlapping cluster fragments $c_{2}=\{E, F\}$ and $c_{3}=$ $\{E\}$. In this case since $c_{3} \subseteq c_{2}$, these clusters can be combined during the pruning step. Thus, we merge any overlapping fragments in the cluster list. Case 2: the cluster does not meet the null storage threshold. Thus, it is partitioned until it meets the null storage threshold. The partitioning process repeatedly removes the property $p$ from the cluster that causes the most null storage until it meets the null threshold. Removing $p$ maximally reduces the null storage in one iteration. Further, support for clusters is monotonic: given two clusters $c_{1}$ and $c_{2}, c_{1} \subseteq c_{2} \Leftarrow \operatorname{support}\left(c_{1}\right) \geq \operatorname{support}\left(c_{2}\right)$. Thus, the partitioned cluster will still meet the given support threshold. After removing $p$, two cases are considered. Case $2 a$ : $p$ exists in a lower-support cluster. Thus, $p$ has a chance of being kept in a n-ray table. Case $2 b$ : $p$ does not exist in a lower-support cluster. This is the worst case, as $p$ must be stored in a binary table. Once the cluster is partitioned to meet the null threshold, it is considered a table and all lower-support clusters with overlapping properties are pruned.

Example. From our running example in Figure 2, two clusters would be passed to the partitioning phase: $\{P 1, P 2, P 3, P 4\} \quad$ and $\{P 1, P 2, P 5, P 6\}$. The cluster $\{P 1, P 2, P 3, P 4\}$ has the highest support value (as given in Figure 2 (b)), thus it is handled first. Since this cluster does not meet the null threshold (as given in Figure 2 (c)) the cluster is partitioned (Case 2) by removing the property 


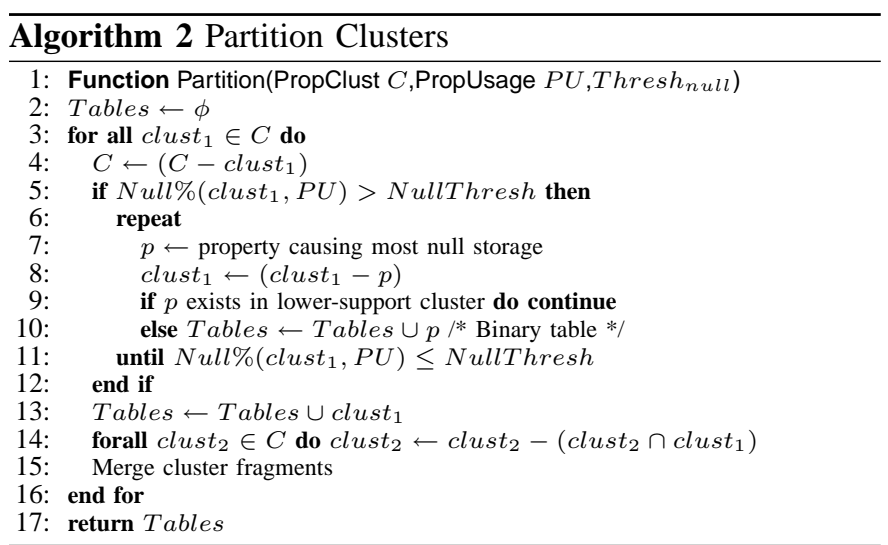

that causes the most null storage, $P 2$, corresponding to the property with minimum usage in the property usage list in Figure 2 (a). Since $P 2$ is found in the lower-support cluster $\{P 1, P 2, P 5, P 6\}$ (Case $2 a$ ), it has a chance of being kept in an n-ary table. Removing $P 2$ from $\{P 1, P 2, P 3, P 4\}$ creates the cluster $\{P 1, P 3, P 4\}$ that falls below the null threshold of $20 \%$ (as given in Figure 2 (d)), thus it is considered a final table. Since $\{P 1, P 3, P 4\}$ and $\{P 1, P 2, P 5, P 6\}$ contain overlapping properties, $P 1$ is then pruned from $\{P 1, P 2, P 5, P 6\}$, creating cluster $\{P 2, P 5, P 6\}$. Since cluster $\{P 2, P 5, P 6\}$ also falls below the null threshold (as given in Figure 2 (d)), it would be added to the final table list in the next iteration. Finally, Figure 2 (e) gives the final schema with the union of tables returned from both phases.

Algorithm. Algorithm 2 gives the psuedocode for the partitioning phase, taking as arguments the list of property clusters $(C)$ from Phase I, sorted in decreasing order by support value, the property usage list $(P U)$, and the null threshold value $\left(\right.$ Thresh $\left._{\text {null }}\right)$. The algorithm first initializes the final table list Tables to empty (Line 2 in Algorithm 2). Next, it traverses each property cluster clust $_{1}$ in list $C$, starting at the cluster with highest support (Line 2 in Algorithm 2). Next, clust $_{1}$ is removed from the cluster list $C$ (Line 2 in Algorithm 2). The algorithm then checks that clust $_{1}$ meets the null storage threshold (Line 2 in Algorithm 2). If this is the case, it considers clust $_{1}$ a final table (i.e., Case 1), and all lower-support clusters with properties overlapping clust $_{1}$ are pruned and cluster fragments are merged. (Lines 2 to 2 in Algorithm 2). If clust $_{1}$ does not meet the null threshold, it must be partitioned (i.e., Case 2). The algorithm finds property $p$ causing maximum storage in clust $_{1}$ (corresponding to the minimum usage count for clust $_{1}$ in $P U$ ) and removes it. (Lines 2 and 2 in Algorithm 2). If $p$ exists in a lowersupport cluster (i.e., Case 2a), iteration continues, otherwise (i.e., Case 2b) $p$ is added to Tables as a binary table (Lines 2 and 2 in Algorithm 2). Partitioning continues until clust $_{1}$ meets the null storage threshold (Line 2 in Algorithm 2). When partitioning finishes, the algorithm considers clust $_{1}$ a final table, and prunes all lower-support clusters of properties overlapping with clust $_{1}$ while merging any cluster fragments (Lines 2 to 2 in Algorithm 2).
$<$ Book1, Auth, Smith> $<$ Book1, Auth, Jones $>$ $<$ Book1, Date, 1998>

(a) RDF Triples

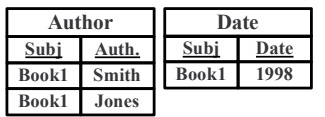

(c) Binary Tables

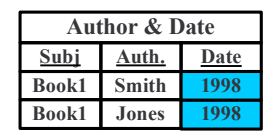

(b) N-ary Table
Fig. 3. Multi-Valued Attribute Example

\section{IMPORTANT RDF CASES}

We now outline two cases for RDF data important to schema creation. The first case deals with storage of multi-valued properties. The second case covers refification.

\section{A. Multi-Valued Properties}

Thus far, only single-valued properties have been considered for storage in n-ary tables. We now propose a method to handle multi-valued properties in our framework. For example, Figure 3(b) gives an example of a multi-valued property data for the RDf triples in Figure 3(a). Each property is assigned a redundancy factor $(r f)$, a measure of repetition per subject in the RDF data set. If $N_{b}$ is the total number of subjectproperty baskets, the redundancy factor for a property $p$ is computed as $r f=\frac{P U \cdot \operatorname{count}(p)}{\operatorname{support}(p) \times N_{b}}$. The term PU.count $(p)$ is a count of the actual property usage in a data set, while the term $\operatorname{support}(p) \times N_{b}$ is the usage count of a property if it were single-valued. We note that the property usage table $(P U)$ stores the usage count (including redundancy) of each property in the data set (e.g., in Figure 3(a), PU.count(auth) $=2$ and PU.count $($ date $)=1$ ), while the subject-property basket stores a property defined for a subject only once (e.g., in Figure 3(a) the basket is book $1 \rightarrow\{$ auth, date $\}$ ). For the data in Figure 3(a), the $r f$ value for auth is $2\left(\frac{2}{1 \times 1}\right)$, while for date it is $1\left(\frac{1}{1 \times 1}\right)$. To control redundancy, a user can define a redundancy threshold, that defines the maximum $r f$ value a property can have in order to qualify for storage in an n-ary table. The $r f$ values multiply each other, thus if two multi-valued properties are stored in an n-ary table, the amount of redundancy is $r f_{1} \times r f_{2}$. Properties not meeting the threshold are explicitly disqualified from the clustering and partitioning phases, and stored in a binary table. The example given in Figure 3(c) stores the auth property in a separate binary table, removing redundant storage of the date property. If the redundancy threshold is 1 , multi-valued properties are not stored in n-ary tables.

If multi-valued properties are allowed, null calculation (Section IV-B) changes. Due to space constraints, we outline how the calculation changes using the example in Figure 3(d), where Prop 1 is single-valued (with $r f=1$ ), while Prop 2 and Prop 3 are multi-valued (with $r f=2$ ). The shaded columns of the table represent the property usage for each property if they were single valued (as calculated in the $r f$ equation). Using these usage values, the initial null storage 


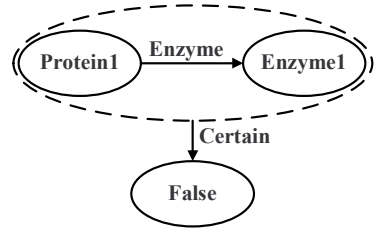

(a) Reification Graph

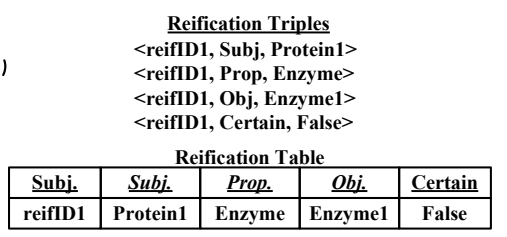

(b) Reification Table

Fig. 4. Reification Example

value for a table can be calculated as discussed in Section IV-B. However, the final calculation must account for redundancy. In Figure 3(d), the table displays three redundancy tiers. Tier 1 represents rows with all three properties defined, thus having a redundancy of 4 (the $r f$ multiplication for Prop 2 and Prop 3). Tier 2 has a redundancy of 2 (the $r f$ for Prop 2). Thus, the repeated null values for the Prop 3 column must be calculated. Tier 3 does not have redundancy (due to the $r f$ value of 1 for Prop 1).

\section{B. Reification}

Reification is an RDF property that allows statements to be made about other RDF statements. An example of reification is given in Figure 4, taken from the Uniprot protein annotation data set [6]. The graph form of reification is given in Figure 4(a), while the RDF triple format is given at the top of Figure 4(b). The Uniprot RDF data stores for each < protein, Enzyme, enzyme > triple information about whether the relationship between protein and enzyme has been verified to exist. This information is modeled by the Certain property, attached as a vertex to the whole $<$ protein, Enzyme, enzyme $>$ triple for the graph representation in Figure 4(a). The only viable method to represent such information in RDF is to first create a new subject ID for the reification statement (e.g., reifIDl in Figure 4(b)). Next, the subject, property, and object of the reified statement are redefined. Finally, the property and object are defined for the reification statement (e.g., certain and false, respectively, in Figure 4(b)). We mention reification as our data-centric method greatly helps query processing over this structure. Notice that for reification a set of at least four properties must always exist together in the data. Thus, our schema creation method will cluster these properties together in an nary table, as given in Figure 4(b). Our framework also makes an exception to allow reification properties subject, property, and object to exist in multiple n-ary tables for each reification edge. This exception means that a separate n-ary table will be created for each reification edge in the RDF data (e.g., Certain in figure Figure 4), Section VI will experimentally test this claim over the real-world Uniprot [6] data set.

\section{EXPERIMENTS}

This section experimentally evaluates our RDF schema creation approach with existing RDF storage methods.

\section{A. Experimental Setup}

We use three real-world data sets in our experiments: DBLP [16], DBPedia [17], and Uniprot [6]. All data sets

\begin{tabular}{|l|c|c|c|}
\hline Statistic & DBLP & DBPedia & Uniprot \\
\hline \# Total Properties & 30 & $19 \mathrm{~K}$ & 86 \\
\hline$\%$ total props stored in binary tables & $40 \%$ & $99.59 \%$ & $69 \%$ \\
\hline$\%$ total props stored in n-ary tables & $60 \%$ & $0.41 \%$ & $31 \%$ \\
\hline \# Multi-Val Properties & 4 & 6080 & 35 \\
\hline Min $r f$ value for multi-val properties & 3.4 & 4 & 1.2 \\
\hline$\%$ multi-val prop stored in n-ary tables & $0 \%$ & $0 \%$ & $17 \%$ \\
\hline
\end{tabular}

(a) Schema Breakdown

\begin{tabular}{|l|c|c|c|c|c|c|}
\hline Data Set & Binary & 3-ary & 4-ary & 5-ary & $(6+)$-ary & Total \\
\hline DBLP & 12 & 2 & 6 & 4 & 6 & 30 \\
\hline DBPedia & 18922 & 8 & 6 & 8 & 56 & $19 \mathrm{~K}$ \\
\hline Uniprot & 60 & 4 & 9 & 8 & 5 & 86 \\
\hline
\end{tabular}

(b) Table Distribution (by Property)

Fig. 5. Data Centric Schema Tables

contain more than 10M triples; specific details for these data can be found in our companion technical report [27]. To create data-centric tables, the support parameter was set to $1 \%$ (a generally accepted default value [28]), the null threshold value was set to $30 \%$, and the redundancy threshold was set to 1.5. Figure 5(a) gives the breakdown of the percentage of all properties for each data set that are stored in n-ary tables or binary tables (rows 1-3). This table gives the number of multi-valued properties in each data set (row 4), along with the minimum redundancy factor from all these properties (row 5). Only the Uniprot data set had multi-valued properties that met the redundancy threshold of 1.5 , thus six of these properties $(17 \%)$ were kept in n-ary tables (row 6). Figure 5(b) gives the table type (binary or n-ary) and the distribution of properties stored in each table type.

The experimental machine used is a 64-bit, 3.0 GHz Pentium IV, running Ubuntu Linux with 4Gbytes of memory. Our schema creation module was built using $\mathrm{C}++$, and integrated with the PostgreSQL 8.0.3 database. We implemented a triple-store similar to many RDF storage applications (e.g., see [7], [9], [11], [12]), which is a single table containing three columns corresponding to an RDF subject, property, and object. We implemented the decomposed storage method by allowing each table to correspond to a unique property in the RDF dataset. Our data-centric approach built both n-ary and binary tables according to the structure of each data set. Indepth implementation details (e.g., indexing) are found in our companion technical report [27].

\section{B. Experimental Evaluation}

This section provides performance numbers for a set of queries based on previous benchmarks for Uniprot [10] and DBPedia [29]. Since the benchmarks were originally designed for their respective data, we first generalize the query in terms of its signature, then give the specific query for each data set. For each query, Figure 6 gives the query runtimes (in seconds) for each of the three storage approaches: triple-store, decomposed storage model (DSM), and our proposed datacentric approach. All times given are the average of ten runs, with the cache cleared between runs.

1) Query 1: Predetermined props/all subjects: Query 1 (Figure 6(a)) asks about a predetermined set of RDF properties. The general signature of this query is to select all records 


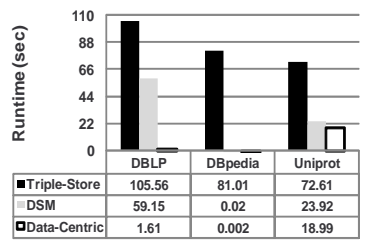

(a) Query 1

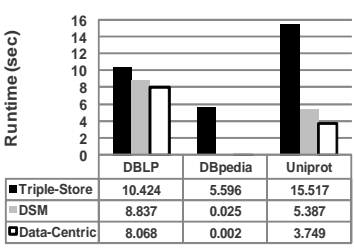

(b) Query 2

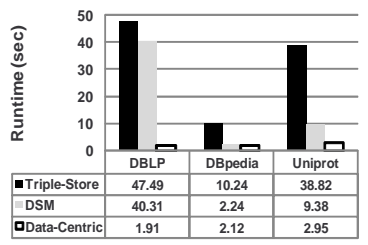

(c) Query 3

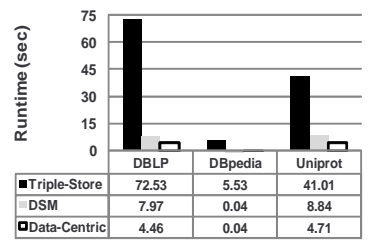

(d) Query 4

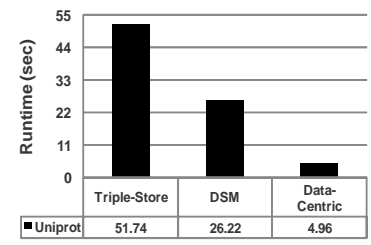

(e) Query 5

Fig. 6. Queries

for which certain properties are defined. For specific data set queries (along with SQL), the reader is encouraged to see our companion technical report [27]. Overall, the data-centric approach shows better relative runtime performance for Query 1. Interestingly, the data-centric approach showed a factor of 65 speedup over the triple-store for the DBLP query, and a factor of 36 speedup over the decomposed approach. The DBLP data is relatively well-structured, thus, our data-centric approach stores a large number of properties in n-ary tables. In fact, the data-centric approach involved a single table access and no joins, while the triple-store and decomposed approach both used six table accesses and five self-joins. Similarly, the data-centric approach shows modest speedup for the DBPedia and Uniprot data sets again due to the decreased table accesses and joins. For instance the data-centric approach required five table accesses in the Uniprot query and four subject-to-subject joins, compared to six table accesses and five joins for the triple-store and decomposed approaches.

2) Query 2: Single subject/all defined properties: Query 2 (Figure 6(b)) involves a selection of all defined properties for a single RDF subject (i.e., a single record). For specific data set queries (along with SQL), the reader is encouraged to see our companion technical report [27]. For DBLP, this query accesses $13 \mathrm{RDF}$ properties. The decomposed and triplestore approach involved 13 table accesses, while the datacentric approach involved nine. The performance between the decomposed and our data-centric approaches is similar in this case, due to the fact that some tables in the data-centric approach contained extraneous properties, meaning some stored properties were not used in the query. For DBPedia, the query accesses 23 RDF properties. The data-centric and decomposed approaches exhibit a similar relative performance to the triplestore with sub-second runtimes. However, the data-centric approach accessed a total of 17 tables, compared to the 23 needed by the decomposed and triple-store approaches. For Uniprot, this query accesses 15 RDF properties. The decomposed and triple-store approach involved fifteen table accesses along with fourteen subject-to-subject joins. Meanwhile, the data-centric approach involved 11 table accesses generating 10 subject-tosubject joins.

3) Query 3: Administrative query: Query 3 (Figure 6(c)) is an administrative query asking about date ranges for a set of recently modified RDF subjects in the data set. The general signature of this query is a range selection over dates. For specific data set queries (along with SQL), the reader is encouraged to see our companion technical report [27]. The data-centric approach shows better relative performance to that of the other schema approaches. Again, for the well-structured
DBLP data, data-centric approach stored all query properties in a single table, causing a factor of 24 speedup over the triple-store, and a factor of 21 speedup over the decomposed approach. This performance is due to the data-centric approach requiring a single table access, with all five queried properties clustered to a single table. Meanwhile, both the the triple-store and decomposed approaches required separate table accesses for the range query and joins. The data-centric approach also shows good speedup for the semi-structured Uniprot data.

4) Query 4: Predetermined props/spec subjects: Query 4 (Figure 6(d))retrieves a specific set of properties for a particular set of RDF subjects. The general signature of this query is a selection of a set of RDF subjects (using the IN operator). For specific data set queries (along with SQL), the reader is encouraged to see our companion technical report [27]. Again, the data-centric approach shows better overall performance to that of the other schema approaches. For the Uniprot and DBLP, the data-centric approach shows good speedup over the triple-store, and a 1.8 speedup over the decomposed approach. The data-centric approach required only two table accesses and one join for the Uniprot data, and a single table access for the DBLP data, compared to four and five table accesses, respectively, for the other storage methods. The performance was similar for the data-centric and decomposed methods over the DBPedia data, as both queries accessed all binary tables.

5) Query 5: Reification: Query 5 (Figure 6(e)) involves a query using reification. For this query, only the Uniprot data set is tested, as it is the only experimental data set that makes use of reification. The query here is to display the top hit count for statements made about proteins. In the Uniprot dataset, this information is stored as reified data with the the object property corresponding to a protein identifier, and the hit count modeled as the hits property. The large difference in performance numbers here is mainly due to the table accesses needed by both the decomposed and triple-store to reconstruct the statements used for reification. Our data-centric approach involved a single table access with no joins, due to the fact that the reification structure being clustered together in n-ary tables. Our approach shows a speedup of 5.29 and 10.44 over the decomposed and triple-store approaches.

6) Relative Speedup: Figure 7(a) gives the relative speedup for the data-centric approach over the triple-store approach for each query and data set, while Figure 7(b) gives the same speedup data over the decomposed approach. The DBLP data set is well-structured, and our data centric approach showed superior speedup for queries 1 and 3 over the DBLP data as it clustered all related data to the same table. Thus, the queries were answered with a single table access, compared to multiple accesses and joins for the triple-store and decomposed 


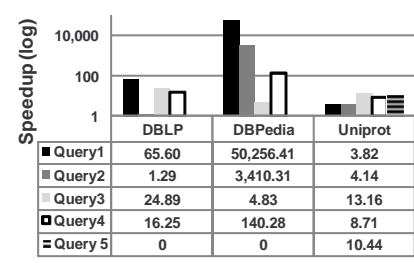

(a) Speedup over Triple Store

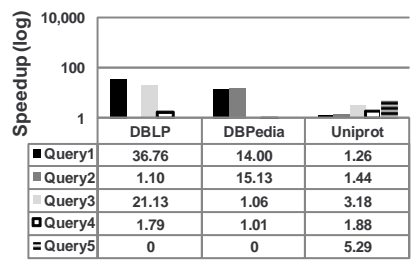

(b) Speedup over DSM
Fig. 7. Relative Speedup

approaches. For DBPedia queries 1 and 2, the data-centric approach showed speedup over the decomposed approach due to accessing the few n-ary tables present to store this data. However, this data was mostly semi-structured, thus queries 3 and 4 showed similar performance as they involved the same table structure. The speedup over the triple-store for DBPedia was superior as queries using the data-centric approach involved tables (1) with smaller cardinality and (2) containing, on average, only the properties necessary to answer the queries, as opposed to the high-selectivity joins used by the large triple-store. Our data-centric approach showed a moderate speedup performance for the Uniprot queries due to two main factors: (1) some data-centric tables contained extraneous properties and multi-valued attributes that caused redundancy, and (2) the semi-structured nature of the Uniprot data set led to a similar number of relative joins and table accesses.

\section{CONCLUSION}

This paper proposed a data-centric schema creation approach for storing RDF data in relational databases. Our approach derives a basic structure from RDF data and achieves a good balance between using n-ary tables (i.e., property tables) and binary tables (i.e., decomposed storage) to tune RDF storage for efficient query processing. First, a clustering phase finds all related properties in the data set that are candidates to be stored together. Second, the clusters are sent to a partitioning phase to optimize for storage of extra data in the underlying database. We compared our data-centric approach with state-of-the art approaches for RDF storage, namely the triple store and decomposed storage, using queries over three real-world data sets. The data-centric approach shows large orders of magnitude performance improvement over the triple store, and speedup factors of up to 36 over the decomposed approach.

\section{REFERENCES}

[1] "World Wide Web Consortium (W3C): http://www.w3c.org."

[2] "W3C Semantic Web Activity: http://www.w3.org/2001/sw/.",

[3] D. Kotzinos, S. Pediaditaki, A. Apostolidis, N. Athanasis, and V. Christophides, "Online curriculum on the semantic Web: the CSDUoC portal for peer-to-peer e-learning," in $W W W, 2005$.

[4] J. S. Jeon and G. J. Lee, "Development of a Semantic Web Based Mobile Local Search System," in $W W W, 2007$.

[5] X. Wu, L. Zhang, and Y. Yu, "Exploring social annotations for the semantic web," in $W W W, 2006$.

[6] "Uniprot RDF Data Set: http://dev.isb-sib.ch/projects/uniprot-rdf/."

[7] S. Alexaki, V. Christophides, G. Karvounarakis, D. Plexousakis, and K. Tolle, "The ICS-FORTH RDFSuite: Managing Voluminous RDF Description Bases," in SemWeb, 2001.

[8] D. Beckett, "The Design and Implementation of the Redland RDF Application Framework," in $W W W, 2001$.
[9] J. Broekstra, A. Kampman, and F. van Harmelen, "Sesame: A Generic Architecture for Storing and Querying RDF and RDF Schema," in ISWC, 2002.

[10] E. I. Chong, S. Das, G. Eadon, and J. Srinivasan, "An Efficient SQLbased RDF Querying Scheme," in $V L D B, 2005$.

[11] S. Harris and N. Gibbins, "3store: Efficient bulk rdf storage," in PSSS, 2003.

[12] L. Ma, Z. Su, Y. Pan, L. Zhang, and T. Liu, "Rstar: an rdf storage and query system for enterprise resource management," in CIKM, 2004.

[13] K. Wilkinson, "Jena Property Table Implementation," in SSWS, 2006.

[14] J. L. Beckmann, A. Halverson, R. Krishnamurthy, and J. F. Naughton, "Extending rdbmss to support sparse datasets using an interpreted attribute storage format," in ICDE, 2006.

[15] D. Abadi, A. Marcus, S. R. Madden, and K. Hollenbach, "Scalable Semantic Web Data Management Using Vertical Partitioning," in VLDB, 2007.

[16] B. Aleman-Meza, F. Hakimpour, I. B. Arpinar, and A. P. Sheth, "Swetodblp ontology of computer science publications," Web Semantics: Science, Services and Agents on the World Wide Web, vol. 5, no. 3, pp. 151-155, 2007.

[17] S. Auer, C. Bizer, G. Kobilarov, J. Lehmann, R. Cyganiak, and Z. Ives, "DBpedia: A Nucleus for a Web of Open Data," in ISWC, 2007.

[18] G. P. Copeland and S. N. Khoshafian, "A Decomposition Storage Model," in SIGMOD, 1985.

[19] A. Matono, T. Amagasa, M. Yoshikawa, and S. Uemura, "A Path-Based Relational RDF Database," in $A D C, 2005$.

[20] R. Angles and C. Gutierrez, "Querying rdf data from a graph database perspective," in ESWC, 2005.

[21] S. Agrawal, S. Chaudhuri, and V. R. Narasayya, "Automated selection of materialized views and indexes in sql databases," in $V L D B, 2000$.

[22] S. Agrawal, V. R. Narasayya, and B. Yang, "Integrating vertical and horizontal partitioning into automated physical database design," in SIGMOD, 2004.

[23] S. B. Navathe and M. Ra, "Vertical partitioning for database design: A graphical algorithm," in SIGMOD, 1989.

[24] S. Papadomanolakis and A. Ailamaki, "Autopart: Automating schema design for large scientific databases using data partitioning," in SSDBM, 2004.

[25] R. Agrawal and R. Srikant, "Fast Algorithms for Mining Association Rules," in VLDB, 1994.

[26] D. Burdick, M. Calimlim, and J. Gehrke, "MAFIA: A Maximal Frequent Itemset Algorithm for Transactional Databases," in ICDE, 2001.

[27] J. J. Levandoski and M. F. Mokbel, "RDF Data-Centric Storage," University of Minnesota, Tech. Rep. UM-CS-TR-9999, 2009.

[28] R. Agrawal and J. Kiernan, "An Access Structure for Generalized Transitive Closure Queries," in ICDE, 1993.

[29] "RDF Store Benchmarks with DBpedia: http://www4.wiwiss.fu-berlin.de/benchmarks-200801/." 